\title{
Impacto y productividad de las publicaciones latinoamericanas sobre Matemática Educativa
}

\author{
Aida María Torres-Alfonso \\ María Josefa Peralta-González \\ Anamarys Toscano- Menocal \\ Universidad Central "Marta Abreu" de Las Villas - UCLV, Cuba
}

ANALYSIS

\begin{abstract}
Resumen
Hace poco más de un cuarto de siglo se han estado realizando, con frecuencia anual las Reuniones Latinoamericanas de Matemática Educativa (RELME) y los trabajos presentados en el evento, luego de una revisión por pares de expertos, se edita el ALME (Acta Latinoamericana de Matemática Educativa). Se presenta un análisis cualitativo del impacto de las publicaciones registradas en ALME en el período comprendido entre los años 2000 y 2009. Profundizando en indicadores de producción científica, indicadores de impacto o influencia: que aportaron el uso que la comunidad científica hace de estos resultados a partir del recuento de citas recibidas por los trabajos publicados en ALME en la propia publicación; complementado por un análisis de citas, según el Google Académico y los indicadores de colaboración que mostraron la tipología de colaboración existente en esta comunidad científica.
\end{abstract}

Palabras claves:

Producción científica ; Matemática educativa ; Indicadores cienciométricos ; Análisis de citas ; Colaboración

\section{Impact and productivity of Latin American publications about mathematics education}

\begin{abstract}
More than a century room recently they have been carrying out, frequently annual Educational Mathematics Latin American Meetings (RELME) and the works presented in the event, after a revision for even of experts, the ALME is published (Latin American Mathematics Education Act). We present a qualitative analysis of the impact of publications recorded in ALME in the period between 2000 and 2009. They use scientific production indicators, indicators of impact or influence: who provided the scientific community use made of these results based on the count of citations received by papers published in ALME in the publication itself, supplemented by an analysis of citations according to Google Scholar and collaboration indicators that showed the type of existing collaboration in the scientific community.
\end{abstract}

Keywords

Scientific production ; Mathematics education ; Scientometrics indicators ; Citation analysis ; Collaboration

\section{Introducción}

Uno de los aspectos fundamentales que caracterizan la actividad científica son las publicaciones científicas que resultan de dicha actividad. En el sistema científico, la tarea de evaluar los nuevos conocimientos y los resultados de la investigación tiene una importancia capital. La ciencia es evaluación y esta a su vez es un proceso social por lo que la forma de llevarla a cabo es determinante para sus resultados. Por tanto el análisis del comportamiento de la actividad científica posibilita conocer el rendimiento y comprobar su impacto en la sociedad. 
Los estudios métricos de la información como disciplina, está conformada por el conjunto de disciplinas científicas las cuales contienen un conjunto de métodos y modelos matemáticos y estadísticos orientados a la medición de diferentes objetos estudios de las Ciencias de la Información (Arencibia y Moya, 2008). En tal sentido las disciplinas métricas más trabajadas en la literatura científica son: la bibliometría, la cienciometría, la informetría y la bibliotecometría, aunque en los últimos tiempos los estudios métricos han estado mayormente dirigidos a la Web y algunas denominaciones conceptuales han estado adecuándose a este medio de comunicación: Internet.

Cada una de estas disciplinas presenta sus objetos y alcances en la práctica informativa, sin embargo el estudio que se presenta, conceptualmente parte de la bibliometría, precisamente porque su unidad de análisis en principio son las publicaciones o trabajos presentados en ALME en forma de referencias bibliográficas, por lo que algunos de los indicadores iniciales del estudio son derivados del recuento o sumatoria de variables (autores, años, países, instituciones) que se encuentran en dichos trabajos. Sin embargo el trabajo va más allá, utiliza indicadores Webométricos referidos a las citas recibidas por ALME en recursos web como el Google Académico, lo cual se traducen el nivel de impacto real que presentan los trabajos publicados por ALME en este medio.

Un aspecto novedoso de este estudio es brindar a la comunidad de investigadores latinoamericanos información valiosa que le permita establecer relaciones de trabajo y proyectos docentes e investigativos en un futuro. Hasta el presente ALME no contaba con un estudio métrico que mostrara el comportamiento de la visibilidad e impacto de las publicaciones que en ella se recogen. A la luz de lo antes expuesto el presente trabajo tiene como objetivo general describir la visibilidad e impacto de las publicaciones científicas de ALME en el período 2000-2009.

Constituyendo por tanto, un punto de partida para futuras investigaciones, ya que no se recogen todos los indicadores bibliométricos, por ejemplo, no se realiza ningún análisis en este trabajo acerca de las palabras claves y temáticas de interés, así como el consumo de información mediante el análisis de referencias de esta publicación.

\section{Métodos y técnicas}

Se aplicaron:

- Método del nivel teórico: Llos relativos a la revisión documental para la introducción del trabajo y contextualización de las disciplinas métricas. El análisis y la síntesis de contenido para elaborar los contenidos del trabajo en función del tipo de publicación. El método inductivo-deductivo, el cual permite la aplicación de una metodología general de los estudios métricos de la información al estudio del comportamiento de indicadores métricos al ALME.

- Métodos del nivel empírico: El análisis documental para la fundamentación del tema. El método bibliométrico como método propio de las Ciencias de la Información que incluyen sus propios modelos y métodos matemáticos que constituye en este trabajo el procedimiento fundamental.

Las técnicas aplicadas se introducen en la aplicación bibliométrica en cada uno de los procedimientos que se describen a continuación:

\subsection{Caracterización de ALME}

El Comité Latinoamericano de Matemática Educativa (CLAME) incluye investigadores y profesores de reconocido nivel en el área de la Matemática Educativa de Latinoamérica, y algunos de sus propósitos fundamentales; como lo es el de aunar acciones e intereses comunes para fomentar investigaciones de calidad, la difusión de la matemática educativa de una manera que sea posible mejorar la calidad de la enseñanza así como brindar un marco en el que puedan relacionarse las personas que comparten este interés común; se han materializado con la realización anual de la Reunión Latinoamericana de Matemática Educativa (RELME), con una historia ininterrumpida de 25 años. 
Se consolida entonces ALME como una publicación con arbitraje, editada en forma digital y que constituye de conjunto con RELIME material obligado de referencia para maestros, profesores y estudiantes de posgrado dentro y fuera de la región.

\subsection{Búsqueda y recuperación de la información}

Se utilizaron como fuentes de información las revistas correspondientes a los eventos RELME que se desarrollan anualmente en Latinoamérica las cuales están publicadas a texto completo en la página oficial de CLAME: http://www.clame.org.mx/alme.htm. Estas revistas no están alojadas en ninguna base de datos, ni regional ni internacional, solo están a disposición de la comunidad científica de Matemática Educativa a través del sitio del CLAME, por lo que se conformó una base de datos intencionada con la normalización de los datos de los 1357 artículos de este periodo.

Como fuente de información secundaria se utilizó el Google Académico, para extraer los datos que pudieran faltar en el completamiento de las referencias de dichas publicaciones así como en la recuperación de los datos de las citas recibidas por ALME en el Google Académico.

\subsection{Búsqueda, procesamiento y representación de la información}

A través del software Publish or Perish se recuperaron y analizaron las citas seleccionadas entre el período 20002009 (Harzing, 2007). Se normalizaron los nombres de los autores y otras variables a medir, a partir la creación de una base de datos de forma manual en el EndNote, para evitar sesgos en la cuantificación de los mismos. Se procesaron un total de 1357 registros resultantes de la búsqueda realizada. Se utilizaron como herramientas el Microsoft Excel para el procesamiento de los datos que permitió identificar los pasos necesarios para realizar cálculos mediante fórmulas y reconocer la información que necesita el asistente de gráficos en cada paso para la confección del mismo; así como también para establecer las relaciones matriciales entre los autores y las citas de los artículos de ALME en ALME (Andalia, 1999).

Otra de las herramientas utilizadas fue el Pajek, el cual permitió la representación el análisis de las redes confeccionadas, este software libre proporciona representar diferentes relaciones de colaboración y cocitación, que favorecen los análisis de impacto de la revista en la comunidad (Arencibia, 2008).

\begin{tabular}{|c|c|c|}
\hline Tipo de Indicador & Indicador & Variables \\
\hline Producción científica & $\begin{array}{l}\text { Productividad por años, por autores, por países y } \\
\text { regiones. }\end{array}$ & Años, autores y países \\
\hline \multirow{3}{*}{ Visibilidad e impacto } & Citas de ALME en ALME. & \multirow{3}{*}{ Citas } \\
\hline & Artículos más citados por ALME. & \\
\hline & Trabajos más citados en el Google Académico. & \\
\hline \multirow{3}{*}{ Colaboración } & Índice de coautoría. & \multirow{3}{*}{$\begin{array}{l}\text { Países, instituciones y } \\
\text { autores. }\end{array}$} \\
\hline & Cantidad de artículos en colaboración. & \\
\hline & $\begin{array}{l}\text { Tipos de colaboración: interinstitucional nacional, } \\
\text { colaboración internacional, colaboración } \\
\text { intraintitucional }\end{array}$ & \\
\hline
\end{tabular}




\section{Análisis de los resultados}

\subsection{Indicadores de actividad o producción científica de ALME}

\section{a) Productividad por años}

El estudio cuantitativo refleja en primer lugar la existencia de fluctuaciones en la producción científica de ALME (Figura. 1). Existen dos picos en la producción científica en el período estudiado: el año 2000 Argentina 200 artículos y el año 2009 con 188, celebrados en Argentina y México respectivamente. Este fenómeno está dado por ser un evento itinerante y no estar exento de las propias limitaciones que desde el punto de vista económico afrontan los autores para participar en RELME, así también están condicionados los dos picos en la producción científica. Cabe señalar que los restantes años aunque fluctúan, sus valores se comportan en el rango: entre 100 y 150 , lo que pudiera indicar sistematicidad en las presentaciones y publicaciones en la revista.

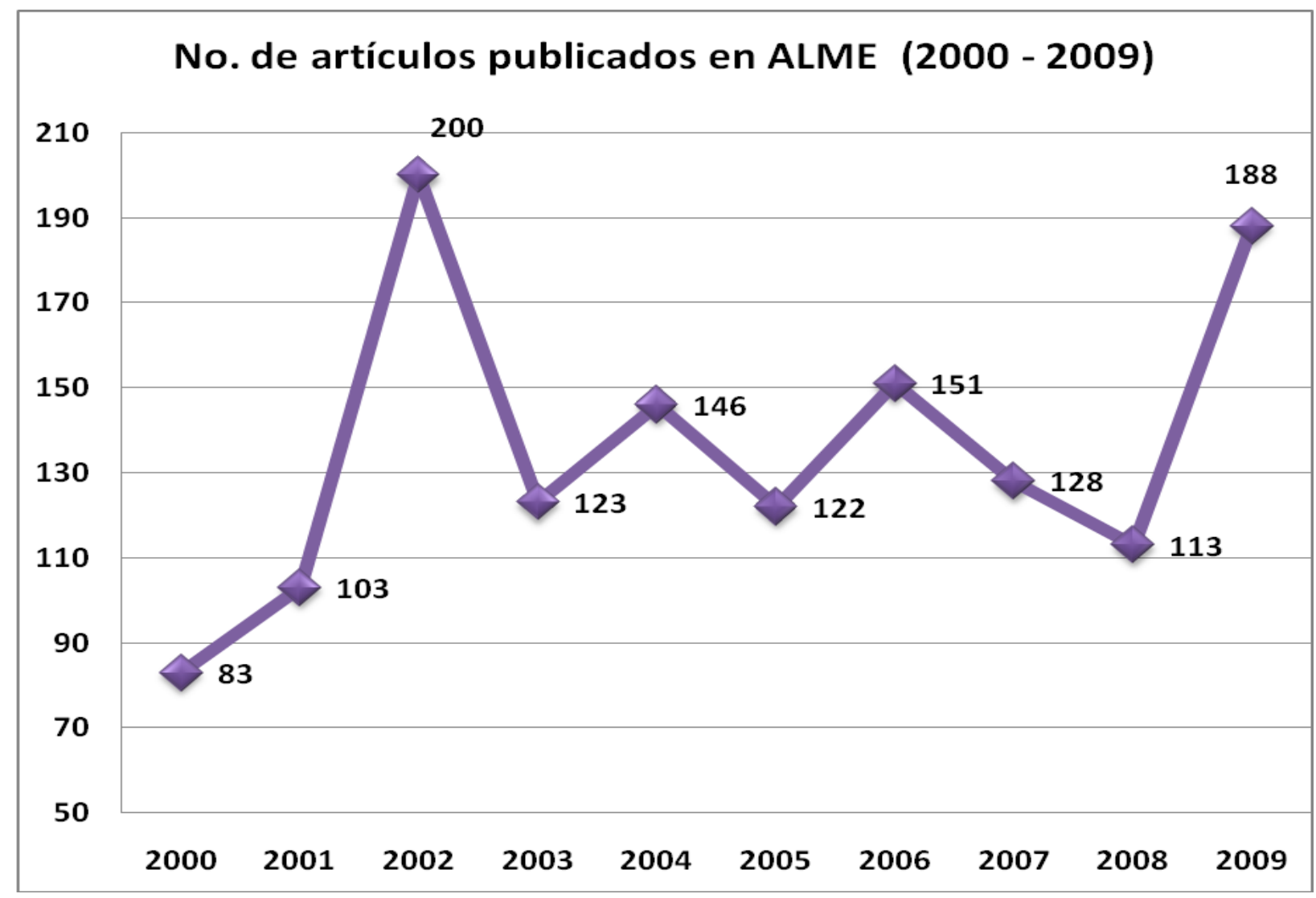

Figura 1 : Evolución de la productividad científica en ALME

Fuente: El autor (2014).

b) Productividad de autores e instituciones por países y regiones

Con el objetivo de realizar un análisis de la productividad científica latinoamericana en ALME, en la Tabla 1 se incluyen la cantidad de artículos, instituciones y autores por países de la región. 
Tabla 1 : Distribución de la productividad latinoamericana de instituciones y autores por países

\begin{tabular}{|l|c|c|c|}
\hline \multicolumn{1}{|c|}{ Países } & Total de artículos & Total Instituciones & Total de Autores \\
\hline México & 581 & 52 & 458 \\
\hline Argentina & 335 & 50 & 417 \\
\hline Cuba & 137 & 21 & 175 \\
\hline Venezuela & 76 & 16 & 54 \\
\hline Uruguay & 43 & 10 & 30 \\
\hline Chile & 41 & 17 & 47 \\
\hline Brasil & 34 & 16 & 42 \\
\hline Colombia & 29 & 14 & 48 \\
\hline Costa Rica & 25 & 4 & 19 \\
\hline Panamá & 20 & 3 & 12 \\
\hline Perú & 14 & 4 & 12 \\
\hline R. Dominicana & 14 & 4 & 11 \\
\hline Guatemala & 7 & 3 & 4 \\
\hline Puerto Rico & 4 & 3 & 1 \\
\hline Honduras & 1 & 1 & 1 \\
\hline Bolivia & 1 & 1 & 1 \\
\hline Haití & 1 & 1 & \\
\hline
\end{tabular}

Fuente: El autor (2014).

Se constata una destacada participación de autores mexicanos, en el caso del resto de los países latinoamericanos y caribeños inciden con mayor presencia Argentina y Cuba en posiciones de avanzada, lo cual expresa la fortaleza de estos países en el tratamiento de la Matemática Educativa como disciplina. En cuanto a la distribución de instituciones representadas en los artículos de ALME en este período se observa que el $50 \%$ se concentran en México y Argentina. Por otra parte, al analizar los tipos de instituciones de estos dos paises que estan representadas en la publicacion analizada, emerge que no sólo es meritorio la alta productividad científica, sino la diversidad de instituciones que participan en la misma, incluyendo centros universitarios, de la enseñanza media, politécnicos, institutos de formación del profesorado y centros educacionales de la enseñanza media y primaria.

Un indicador que incluimos en este estudio, en cuanto a la visibilidad de esta revista digital, por considerarlo significativo, lo es: autores no latinoamericanos, ya que aportan una visión más alla de lo regional sobre este conocimiento. En este sentido se destacan los investigadores españoles, que en el periodo analizado alcanzan la cifra de 35. Otros autores no latinoamericanos tambien contribuyen con su aporte y colaboración, este es el caso de: Estados Unidos (7), Francia (9), Italia (2) y Dinamarca (1).

En el ambito latinoamericano entre los autores mas proliferos, se destacan líderes de la Matematica Educativa en la región, que pertenecen a instituciones donde evidentemente se consolidan en este período diferentes frentes de investigación, como lo son por ejemplo los mexicanos: Ricardo Cantoral, Rosa Maria Farfán y Francisco Cordero del CINVESTAV en el IPN: Instituto Politecnico Nacional, asi como la argentina Cecilia Crespo del Instituto Superior del Profesorado "Dr. Joaquín V. González", el uruguayo Victor Martinez Luaces de la Universidad de la República, el venezolano Andógueni del Instituto Pedagógico de Barquisimeto y el cubano Eugenio Carlos del Instituto Superior Politécnico Jose Antonio Echeverria.

En la Tabla 2 se incluyen el total de artículos de los veinte autores más productivos en este período en ALME, donde ademas se destaca como un frente de investigacion de la region: la Universidad Autónoma de Guerrero. 
Tabla 2 : Autores más productivos en ALME (2000-2009)

\begin{tabular}{|c|c|c|}
\hline Autores & Institución & Total de artículos \\
\hline Cordero, F. & Cinvestav, IPN (México) & 35 \\
\hline Cantoral, R. & Cinvestav, IPN (México) & 34 \\
\hline Farfán, R. M. & Cinvestav, IPN (México) & 30 \\
\hline Arrieta, J. L. & Universidad Autónoma de Guerrero (México) & 24 \\
\hline Crespo, C. & Instituto Superior del Profesorado "Dr. Joaquín V. González" (Argentina) & 24 \\
\hline Martínez-Sierra, G. & Universidad Autónoma de Guerrero (México) & 23 \\
\hline Ferrari, M. & Universidad Autónoma de Guerrero (México) & 21 \\
\hline Buendía, G. & Universidad Autónoma de Chiapas (México) & 19 \\
\hline Lezama, J. & IPN (México) & 19 \\
\hline Anido, M. & Universidad Nacional Rosario (Argentina) & 18 \\
\hline Dolores, C. & Universidad Autónoma de Guerrero (México) & 16 \\
\hline Martínez Luaces, V. & Universidad de la República (Uruguay) & 15 \\
\hline Andógueni, M. & Instituto Pedagógico de Barquisimeto (Venezuela) & 13 \\
\hline Carlos, E. & ISPJAE (Cuba) & 13 \\
\hline De Faria, E. & Universidad de Costa Rica (Costa Rica) & 13 \\
\hline Montiel, G. & IPN (México) & 13 \\
\hline Suárez, L. & IPN (México) & 13 \\
\hline Aparicio, E. & Universidad Autónoma de Yucatán (México) & 11 \\
\hline Camarena, $\mathrm{P}$. & IPN (México) & 11 \\
\hline Ojeda, A. M. & Cinvestav, IPN (México) & 11 \\
\hline
\end{tabular}

Fuente: El autor (2014).

\subsection{Indicadores de impacto basados en el análisis de citas}

Los indicadores de impacto basados en el número de citas muestran el impacto real de una publicación, teniendo en cuenta el reconocimiento explícito de las citas que recibe la misma. Por las características ya descritas de esta publicación el estudio se centró en las citas que ha recibido ALME en el Google Académico y en los artículos recogidos en la propia revista. Se constató en primer lugar el impacto del propio evento para la construcción del conocimiento matemático en los años iníciales del estudio, al encontrarse algunas citas de años anteriores al período estudiado, esto indica continuidad de las investigaciones realizadas anteriormente y el impacto de algunas publicaciones anteriores.

\section{a) Artículos más citados en el Google Académico}

Este indicador permite conocer el impacto real de las publicaciones de ALME en la comunidad internacional. Utilizar el Google Académico como parámetro de comparación resulta más abarcador en el espectro de la comunicación de la ciencia. En este caso se encuentra algo favorable a ALME, la coincidencia de que el autor más citado en esta fuente: Ricardo Cantoral, coincide con el autor más citado dentro de la propia ALME. 
Tabla 3 : Artículos de ALME más citados según el Google Académico

\begin{tabular}{|c|c|l|c|}
\hline Citas & Autores & \multicolumn{1}{|c|}{ Título } & Año \\
\hline 18 & $\begin{array}{l}\text { R Cantoral, } \\
\text { J Molina... }\end{array}$ & Socioepistemología de la Predicción & 2005 \\
\hline 17 & F Cordero & $\begin{array}{l}\text { Lo social en el conocimiento matemático: reconstrucción de argumentos y } \\
\text { significados }\end{array}$ & 2003 \\
\hline 16 & G Montiel & Una caracterización del contrato didáctico en un escenario virtual & 2005 \\
\hline 16 & R Cantoral & Desarrollo del pensamiento y lenguaje variacional, una mirada socioepistemológica & 2004 \\
\hline 15 & M Ferrari & Una visión socioepistemológica. Estudio de la función logaritmo & 2003 \\
\hline 11 & R Cantoral & $\begin{array}{l}\text { Pasado, presente y futuro de un paradigma de investigación en Matemática } \\
\text { Educativa }\end{array}$ & 2000 \\
\hline 7 & $\begin{array}{c}\text { E Carlos } \\
\text { Rodríguez }\end{array}$ & $\begin{array}{l}\text { La superación del profesor de matemática en la Universidad de hoy. Una } \\
\text { experiencia cubana }\end{array}$ & 2000 \\
\hline 7 & F Cordero... & La comprensión de la periodicidad en los contextos discreto y continuo & 2001 \\
\hline 7 & $\begin{array}{c}\text { J Arrieta, G } \\
\text { y cols. }\end{array}$ & Las prácticas sociales como generadoras del conocimiento matemático & 2004 \\
\hline 6 & F Cordero & $\begin{array}{l}\text { El comportamiento periódico de una función como un argumento contextual. La } \\
\text { manifestación del movimiento fuera del instante }\end{array}$ & 2002 \\
\hline
\end{tabular}

Fuente: El autor (2014).

\section{b) Artículos más citados por ALME}

Al realizar la normalización de los datos en el EndNote se constataron 220 artículos de ALME, citados en los 1357 registros correspondientes al período que se analiza, en la tabla IV se muestran los artículos más citados. Es claramente visible el impacto que ha tenido el artículo publicado en el año 2003 por Francisco Cordero: Lo social en el conocimiento matemático: reconstrucción de argumentos y de significados, ha devenido en apoyo a otros 16 artículos presentados en ALME en todos los años desde el 2004 hasta el 2009. Los restantes artículos ubicados en orden descendiente muestran años de citación desde 1998 hasta 2004, por lo que se evidencia la significación que han tenido estos artículos y que han prevalecido en el período estudiado. (Ver Tabla 4).

Tabla 4 : Artículos más citados por ALME

\begin{tabular}{|c|l|c|}
\hline Año & \multicolumn{1}{|c|}{ Artículo de ALME citado } & Total \\
\hline 2003 & $\begin{array}{l}\text { Cordero, F. (2003). Lo social en el conocimiento matemático: reconstrucción de argumentos y de } \\
\text { significados. }\end{array}$ & 16 \\
\hline 2004 & $\begin{array}{l}\text { Cantoral R. (2004) Desarrollo del pensamiento y lenguaje variacional, una mirada } \\
\text { socioepistemológica }\end{array}$ & 9 \\
\hline 2000 & $\begin{array}{l}\text { Cantoral, R. (2000). Pasado, presente y futuro de un paradigma de investigación en Matemática } \\
\text { Educativa. }\end{array}$ & 9 \\
\hline 2005 & Cordero F. (2005). La Socioepistemología en la graficación del discurso Matemático Escolar & 8 \\
\hline 2001 & Cantoral, R. (2001) Sobre la articulación del discurso matemático y sus efectos didácticos & 8 \\
\hline 1998 & $\begin{array}{l}\text { Cantoral, R (1998). La aproximación Socioepistemóngica a la investigación en Matemática } \\
\text { Educativa: El caso del pensamiento y lenguaje variacional }\end{array}$ & 8 \\
\hline 2002 & Crespo Crespo, C. (2002). La noción de infinito a través de la historia & 7 \\
\hline
\end{tabular}

Fuente: El autor (2014).

Análisis de las relaciones que se establecen entre los autores de la comunidad de Matemática Educativa, atendiendo a los trabajos citados y los autores que citan:

En función de valorar el impacto de las investigaciones en el desarrollo de la comunidad de Matemática Educativa se establecieron las relaciones entre sus miembros tomando en consideración un artículo y los autores que con posterioridad lo citan. Por ejemplo, el artículo más citado (Cordero F, 2003) fue citado por 12 autores de manera 
continua durante todo el periodo analizado, en algunos casos por colectivos de autores que desarrollan su investigación en otra institución como es el caso de Jaime Arrieta en la Universidad Nacional Autónoma de Guerrero, que lo cita cuatro veces en diferentes años en artículos publicados en colaboración con otros autores en una línea de investigación que relaciona las prácticas sociales y la modelación; por otra parte Saúl Ezequiel Ramos de la Universidad Nacional Autónoma de Chiapas lo cita en tres ocasiones en diferentes años al estar investigando la epistemología de la matematización de la economía. Por último es de destacar como la continuidad de investigaciones desarrollados por el autor colocan cuatro autocitas en este análisis, pero que fortalecen la influencia de la publicación al ser artículos elaborados por dos o más autores, como es el artículo de Liliana Suarez (2005) en el cual ambos autores exponen los resultados de una importante investigación para la comunidad científica: la Modelación en la Matemática Educativa.

Estamos en presencia sin dudas, de un Frente de Investigación que lidera en Latinoamérica el Cinvestav, del IPN en Ciudad México, al cual pertenece el autor citado y dos de los autores citantes y un cuarto autor que es Eduardo Carrasco, Tesista Magister en el CICATA, del propio IPN: Instituto Politécnico Nacional (Figura 2).

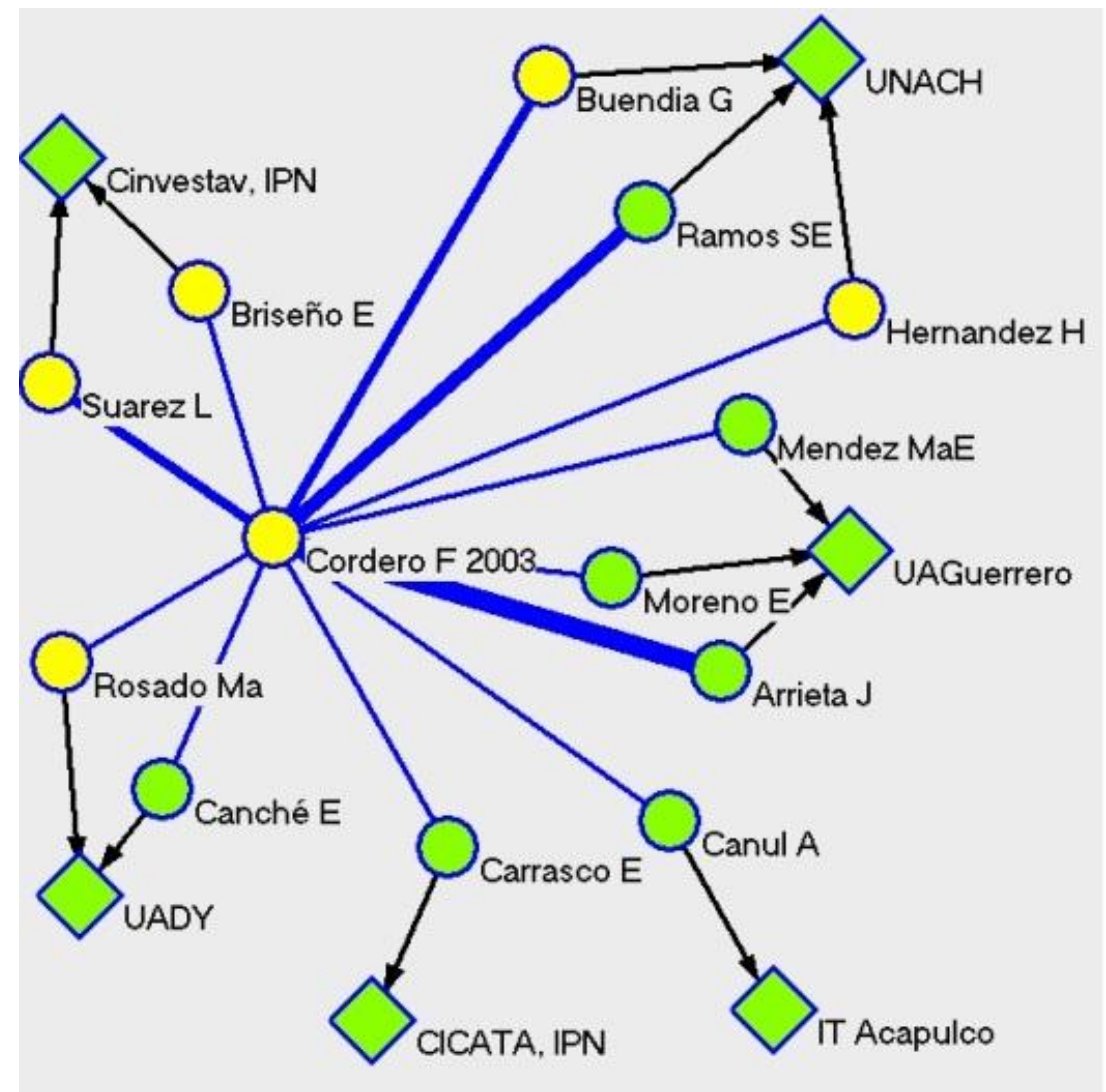

Figura 2 : Impacto del artículo mas citado en ALME Fuente: Cordero (2003).

Otra relación diferente pero de estos dos mismos centros mexicanos, une a los autores Rosa M. Farfán y Gustavo Martínez-Sierra, con sus artículos citados en diferentes años, mostrando como comienzan a publicar y citar juntos acerca de los procesos sociales de generación de conocimiento matemático en el año 2001 y a partir del año 2005 Martínez-Sierra comienza a desarrollar y liderar una línea de investigación en su universidad de origen Universidad Nacional Autónoma de Guerrero, la que busca elaborar explicaciones de los procesos presentes en la articulación de los sistemas conceptuales matemáticos ó procesos de convención matemática, la figura 3 representa esta evolución e impacto de una formación en Educación Matemática, que inicia en el IPN y se consolida en otra institución mexicana. 
Como ejemplo de este impacto, fuera del contexto mexicano la figura 4 muestra la relación que durante el periodo analizado presentan las autocitas que realiza el profesor uruguayo Martínez Luaces, donde un primer momento comienza a publicar solo en la temática de la resolución de problemas y a partir del año 2002 continúa auto citándose, pero a partir de ese momento ya publica en colaboración con otros autores en el campo de la aplicación de los modelos matemáticos.

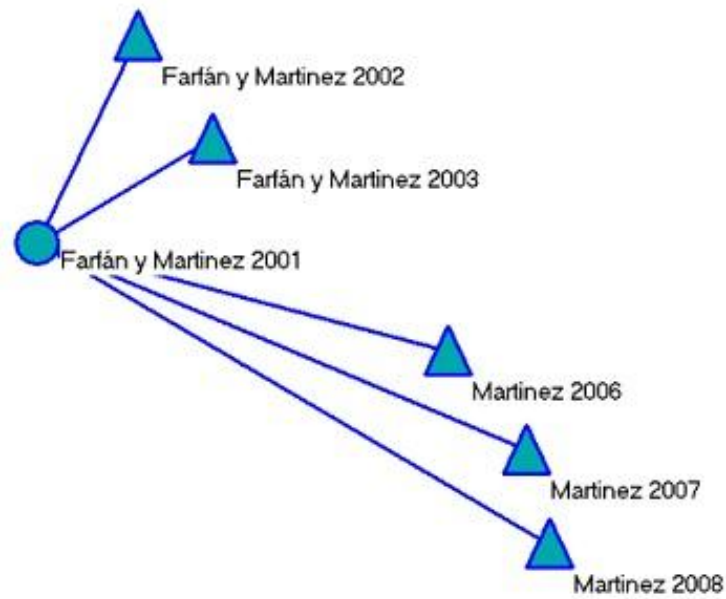

Figura 3 : Evolución de un investigador según un análisis de citas Fuente: El autor (2014).

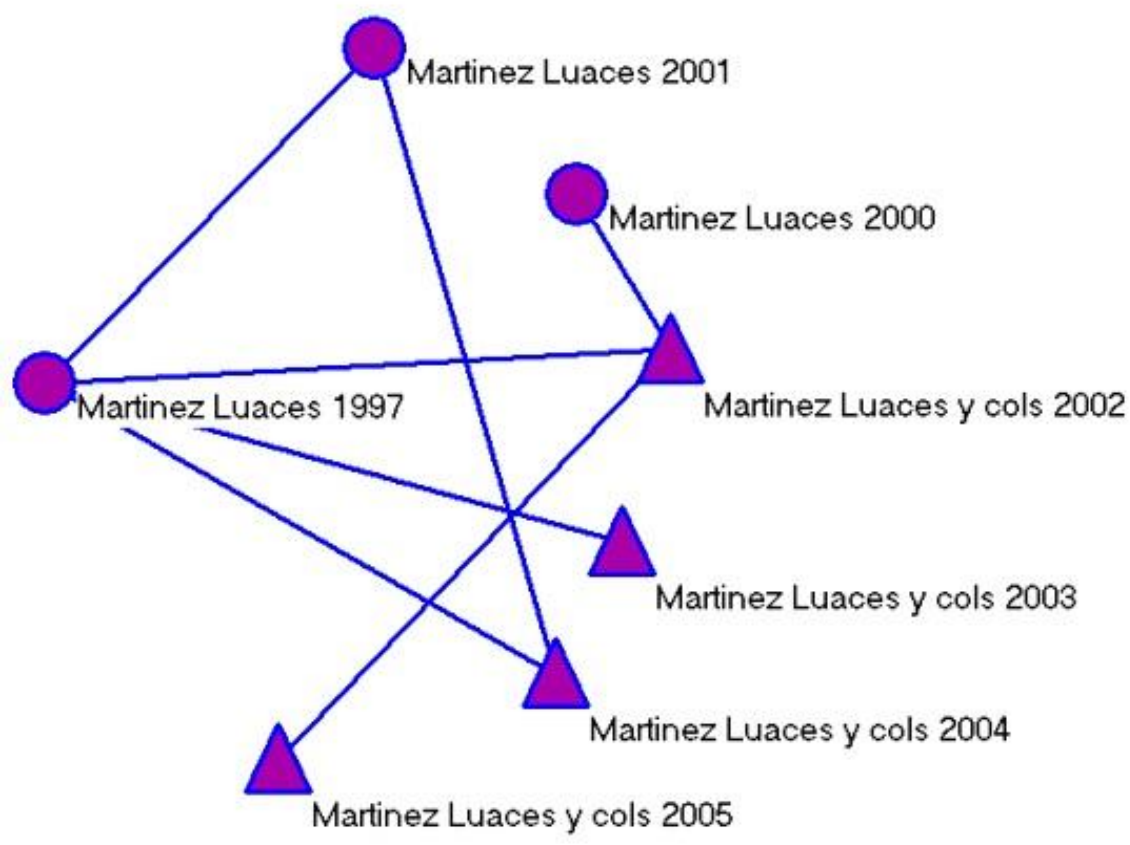

Figura 4 : Análisis del impacto de autocitas en ALME

Fuente: El autor (2014). 


\subsection{Indicadores de colaboración}

\section{a) Índice de coautoría}

El índice de coautoría muestra la colaboración entre autores y se calcula dividiendo autores totales / total de trabajos, en este caso el IC de ALME en el período estudiado es de 2,14. Existe una disminución representativa en este índice en los años 2001 y 2005, reuniones que fueron celebradas en Panamá y México respectivamente y cuyas causales pueden ser motivo de investigaciones posteriores (Figura.5).

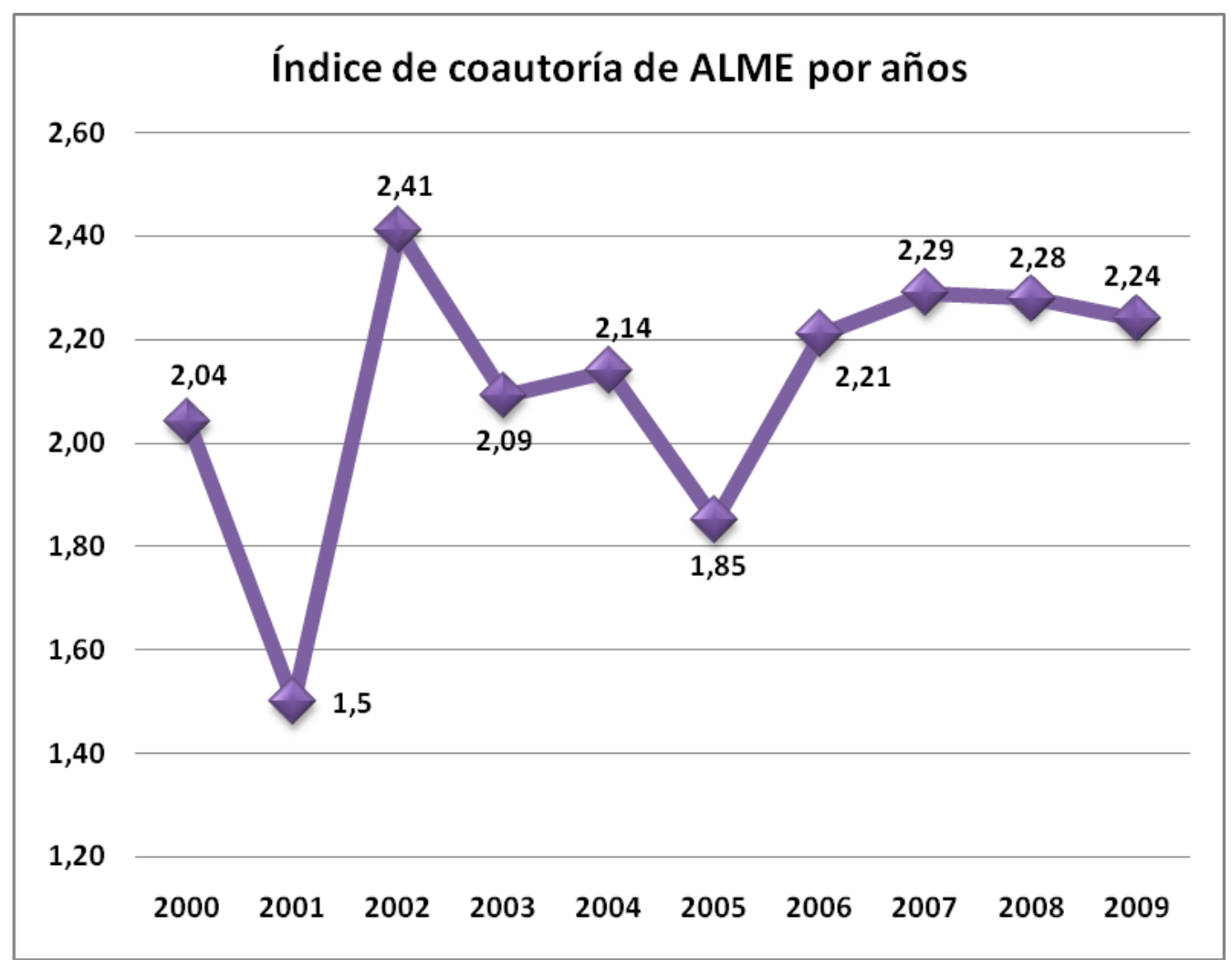

Figura 5 : Índice de coautoría de ALME Fuente: El autor (2014).

\section{b) Proporción de trabajos en colaboración}

En la distribución de los autores de los artículos de ALME según el número de firmas, es alto el número de artículos elaborados en colaboración, es decir, con dos firmas o mas, lo cual ha implicado para las autoras un análisis de los tipos de coautoría presentes en ALME. Constatándose que predomina la colaboración intrainstitucional, ratificando en el área de la Matemática Educativa la fortaleza de grupos de investigación hacia el interior de las propias instituciones y se evidencia una insuficiente colaboración entre instituciones nacionales e internacionales (Figura 6). 


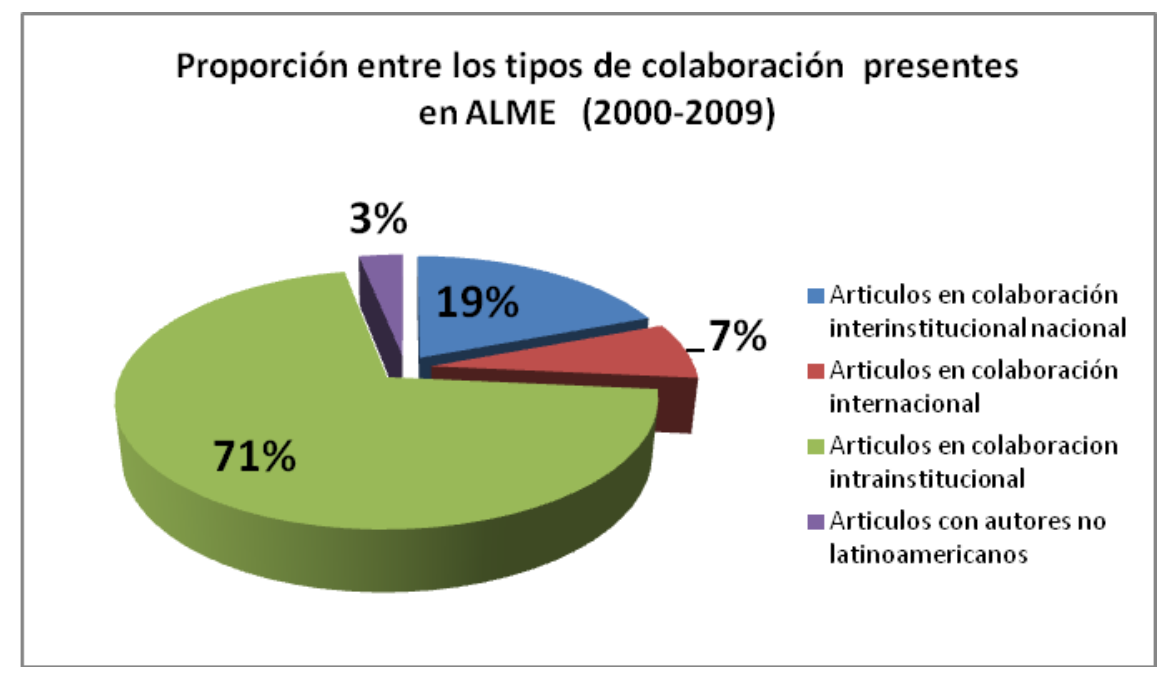

Figura 6 : Tipología de la colaboración en ALME

Fuente: El autor (2014).

\section{c) Colaboración entre países e instituciones}

De un total de 22 países que sus autores publican en ALME, 17 son latinoamericanos y 6 de ellos lo hacen sin colaboración: Puerto Rico, Guatemala, Costa Rica, Honduras, Bolivia y Panamá. Sin embargo presentan un alto índice de colaboración países como Argentina, Brasil, Cuba, México, Uruguay y Chile (Figura 7).

El país no latinoamericano con mayor presencia en ALME en este periodo es España con un total de 23 artículos y de ellos el $65 \%$ en colaboración con países de Latinoamérica. Han estado representadas en este evento 17 instituciones españolas y las de mayor productividad se pueden apreciar en la Tabla 5.

Aun cuando la colaboración interinstitucional es aún baja, existen instituciones que se destacan por su colaboración nacional e internacional, por ejemplo CINVESTAV-IPN (Figura 8).

Tabla 5 : Instituciones españolas con mayor presencia en ALME

\begin{tabular}{|l|c|c|}
\hline \multicolumn{1}{|c|}{ Universidad Española } & Total de artículos & Artículos en Colaboración Latinoamericana \\
\hline Universidad de Granada & 14 & 8 \\
\hline Universitat de Barcelona & 6 & 4 \\
\hline Universidad Autónoma de Barcelona & 4 & 3 \\
\hline Universidad de Jaén & 4 & 1 \\
\hline Universidad Politécnica de Valencia & 3 & 2 \\
\hline
\end{tabular}

Fuente: El autor (2014). 

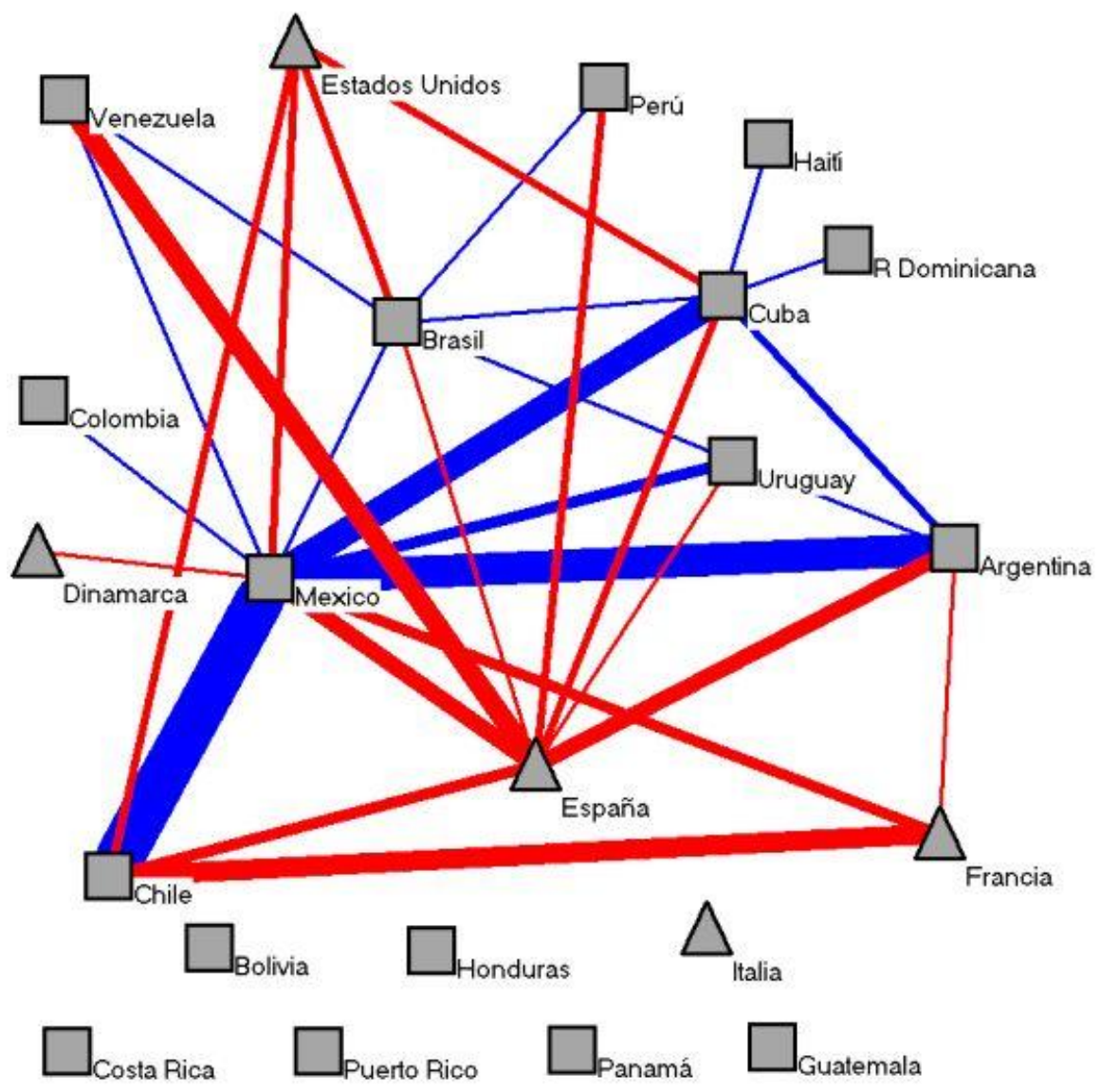

Figura 7 : Colaboración entre países en ALME (2000-2009)

Fuente: El autor (2014).

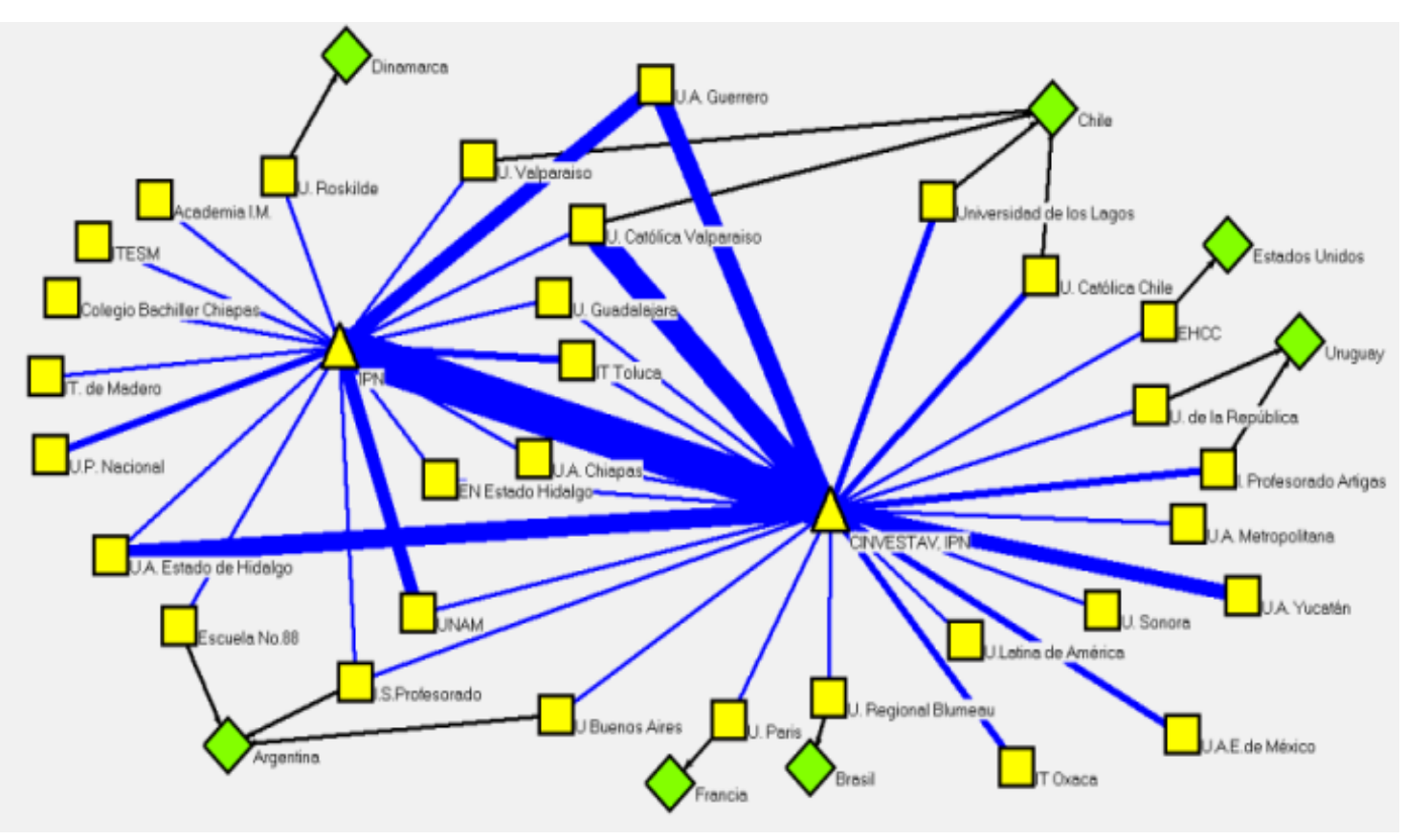

Figura 8 : Colaboración interinstitucional del CINVESTAV-IPN en ALME (2000-2009)

Fuente: El autor (2014). 


\section{Conclusiones}

Las reuniones organizadas por el CLAME: Comité Latinoamericano de Matemática Educativa se han convertido en la posibilidad real de intercambio de experiencias docentes, de exposición de resultados de estudios de postgrados en la región, de consolidación de la teoría de la Matemática Educativa en el contexto latino, y de valoración de las prácticas de la investigación en esta disciplina en América Latina. Desde el inicio de las mismas se ha contado con el interés y la participación de investigadores de Estados Unidos y varios países europeos, entre los que se destaca España.

La institución con mayor aporte científico es el Instituto Politécnico Nacional (IPN), de México, cuestión que solo corrobora la fortaleza que tanto el CINVESTAV, como el CICATA poseen como frentes de investigación consolidados en la región, que propician no solo su participación, sino que promueven la colaboración nacional e internacional como vía de formación de los profesores y de visibilidad de la institución, del país y de la disciplina científica en cuestión.

La importancia de este tipo de estudios métricos en el contexto latinoamericano, particularmente en el área de la Matemática Educativa radica en que aporta elementos objetivos para establecer los frentes de investigación y cuál es el nivel de actualidad y colaboración. Así como permitirá establecer redes temáticas en la región lo que ampliará las posibilidades de proyectos conjuntos y de fortalecer los resultados de las investigaciones que se realicen en función de mayor calidad de la enseñanza y el aprendizaje de la matemática en la región.

Estudios posteriores deberían orientarse a determinar redes de colaboración y la determinación de colegios invisibles, así como análisis de cocitación y de cowords que podrían conformar informaciones relevantes para la aspiración del CLAME de un Observatorio de Matemática Educativa en la región.

\section{Bibliografía}

Andalia, R. (1999). Los análisis de citas en la evaluación de los trabajos científicos y las publicaciones seriadas. $A C I M E D, 7(1)$.

Arencibia, R. (2008). ACIMED en Google Scholar: un análisis de citas de la Revista Cubana de los Profesionales de la Información y Comunicación en la Salud. ACIMED, 18(1).

Arencibia, R. y Moya, F. (2008). La evaluación de la investigación científica una aproximación teórica desde la Cienciometría. ACIMED, 17(4).

Beitía, G. (2001). Algunas tendencias de la Matemática educativa en Latinoamérica. Acta Latinoamericana de Matemática Educativa, vol.14, 14-17.

Campanario, J.M. y Candelario, A. (2010). La influencia de las autocitas en el aumento del factor de impacto en revistas de Ciencias Sociales. Revista española de documentación científica, 33(2), 185-200.

Cañedo, R. (1999). Los análisis de citas en la evaluación de los trabajos científicos y las publicaciones seriadas. $A C I M E D, 7(1)$.

González, M.V. y Molina, M. (2008). La evaluación de la ciencia y la tecnología: revisión de sus indicadores. ACIMED, 18(6).

Harzing, A. Google Scholar: the democratization of citation analysis? [en línea]. Disponible en: http://www.harzing.com/download/gsdemo.pdf [Consultado: 11 de Febrero 2011]

Maz-Machado, A., Bracho-López, R., Torralbo-Rodríguez, M., Hidalgo-Ariza, M. D. y Gutiérrez-Arenas, M. P. (2011). La investigación en Educación Matemática en España: los simposios de la SEIEM. PNA, 5(4), 163-184.

Moya-Anegón, F. y cols. (2004). Cocitación de clases y categorías: Proyecto Atlas de la Ciencia. En: El Estado de la Ciencia. Principales Indicadores de Ciencia y Tecnología Iberoamericanos Interamericanos, cap 3, 1-18. Buenos Aires: RICyT.

Olmeda-Gómez, C. y cols. (2008). Impacto internacional de la investigación y la colaboración científica de las Universidades de Cataluña: 2000-2004. Revista española de Documentación Científica, 31(4), [en línea] Disponible en: http://e-archivo.uc3m.es/handle/10016/10027 [Consultado: 20 de Mayo de 2011].

Zulueta, M., Cabrero, A. y Bordons, M. (1999). Identificación y estudio de grupos de investigación a través de indicadores bibliométricos. Revista española de documentación científica, 22(3), 333-347. 


\section{Datos de los autores}

Aida María Torres-Alfonso

Doctora en Ciencias Pedagógicas y profesora del Centro de Estudios de Educación, Universidad Central "Marta Abreu" de Las Villas.

aidam@uclv.edu.cu

María-Josefa Peralta-González

Magister en Ciencias de la Información y profesora del Departamento de Ciencias de la Información, Universidad Central "Marta Abreu" de Las Villas.

mjosefa@gmail.com

Anamarys Toscano-Menocal

Licenciada en Ciencias de la Información y especialista del Centro de Documentación e Información Científico Técnica - CDICT, Universidad Central "Marta Abreu" de Las Villas.

director3@uclv.edu.cu

Recibido - Received: 2013-12-17

Aceptado - Accepted: 2014-06-30

\section{(oc) EY}

This work is licensed under a Creative Commons Attribution 4.0 United States License.

\section{ULLIS D-Senke}

This journal is published by the University Library System of the University of Pittsburgh as part of its $\underline{D-S c r i b e}$ Digital Publishing Program and is cosponsored by the University of Pittsburgh Press. 